Anuario del Instituto de Historia Argentina, diembre 2018, vol. 18, n² 2, e078. ISSN 2314-257X Universidad Nacional de La Plata

Facultad de Humanidades y Ciencias de la Educación

Centro de Historia Argentina y Americana

\title{
El sistema sanitario durante el neoconservadurismo en Mendoza: problemas y propuestas de cambio entre 1932 y 1943
}

Ivana Hirschegger

CONICET, Argentina

ivanah@mendoza-conicet.gov.ar

Cita sugerida: Hirschegger, I. (2018). El sistema sanitario durante el neoconservadurismo en Mendoza: problemas y propuestas de cambio entre 1932 y 1943 . Anuario del Instituto de Historia Argentina, 18(2), e078. https://doi.org/10.24215/2314257Xe078

Recibido: 11 diciembre 2017 - Aceptado: 22 marzo 2018 - Publicado: 07 diciembre 2018

(c) (1) (2) Esta obra está bajo licencia Creative Commons Atribución-NoComercial-CompartirIgual 4.0 Internacional http://creativecommons.org/licenses/by-nc-sa/4.0/deed.es_AR 


\title{
El sistema sanitario durante el neoconservadurismo en Mendoza: problemas y propuestas de cambio entre 1932 y 1943
}

\author{
The health system during neoconservatism in Mendoza: problems and proposals for change between 1932 and 1943 \\ Ivana Hirschegger \\ CONICET, Argentina \\ ivanah@mendoza-conicet.gov.ar
}

\begin{abstract}
Resumen:
La presente investigación pretende realizar un recorrido por el sistema sanitario mendocino entre los años 1932 y 1943 , es decir, aquella etapa en la cual gobernaron la provincia miembros del Partido Demócrata, y que precedió a la definitiva reorganización del sistema sanitario de carácter centralizado bajo la órbita del Ministerio de Salud Pública de la Provincia, formado en 1950. El hecho de que el sistema sanitario durante el período neoconservador no haya sido estudiado despierta nuestro interés por identificar cuáles fueron los signos centralizadores emergentes, los intentos de reorganización del sistema, y las acciones del Estado para hacer frente a las diferentes problemáticas sanitarias. En definitiva, pondremos el foco en aquellas iniciativas que, si bien cobraron mayor impulso durante los años 40 y 50, tuvieron su génesis en los gobiernos neoconservadores.
\end{abstract}

Palabras Clave: Salud pública, Centralización, Neoconservadurismo, Servicios sanitarios.

\section{Abstract:}

The present investigation intends to make a tour of the Mendoza health system between 1932 and 1943, that is that stage in which the province governed members of the Democratic Party, and which preceded the definitive reorganization of the centralized health system under the orbit of the Ministry of Public Health of the Province, formed in 1950. The fact that the health system during the neoconservative period has not been studied awakens our interest in identifying what were the centralizing signs emerging, the attempts to reorganize the system, and the actions of the State to deal with the different sanitary problems. In this way, it is sought to establish some connections with respect to subsequent governments and to know mainly those initiatives that, originating in the neoconservative governments, were inherited by the Peronists.

KEYWORDS: Public health, Centralization, Neoconservatism, Health services.

\section{INTRODUCCIÓN}

La presente investigación pretende realizar un recorrido por el sistema sanitario mendocino entre los años 1932 y 1943, es decir, aquella etapa en la cual gobernaron la provincia miembros del partido demócrata, y que precedió a la definitiva reorganización del sistema sanitario de carácter centralizado bajo la órbita del Ministerio de Salud Pública de la Provincia, formado en 1950. Esto último significó que el gobierno provincial concentrara la mayor parte de los servicios de salud de la jurisdicción bajo su administración, y ejerciera además un rol predominante en la ejecución de la política sanitaria respecto del gobierno nacional y municipal (Hirschegger, 2010).

La expansión del Estado provincial en materia sanitaria fue un proceso que comenzó a gestarse a finales del siglo XIX, y que a través de los años fue asumiendo nuevas competencias y atribuciones. Durante el lencinismo (1917-1930), a través la Dirección General de Salubridad, el gobierno propuso extender y mejorar los servicios de asistencia médica a los sectores populares, concretando la descentralización geográfica de los servicios sociales a los departamentos de Mendoza. También se avanzó en la creación y ampliación de servicios médico-asistenciales, y en el diseño de una planificación de la atención de la salud para los sectores populares y de asistencia alimentaria para madres y niños. Fue importante también la construcción y ampliación de redes de agua y cloacas en materia de obras sanitarias. Hubo además cambios legislativos que 
otorgaban nuevas funciones al organismo sanitario. Sin embargo, a pesar de los avances, la asistencia médica a la población continuó siendo precaria y la infraestructura insuficiente, por lo que no se pudieron modificar sustancialmente las condiciones de vida de estos sectores en relación con el período conservador (RichardJorba: 2012).

En los años 30, diversos proyectos institucionales en las provincias argentinas, aunque con diversos matices y niveles de ejecución, acrecentaron la capacidad de intervención del Estado en materia sanitaria, algo que puede explicarse en un contexto nacional de cambios en la disciplina y en las corporaciones médicas, de creciente intervención del Estado en el plano social, y de mayor impacto de los organismos y conferencias internacionales en la organización de las políticas sanitarias (Bacolla, 2016). Según los estudios de otras provincias del país, como por ejemplo Córdoba, durante esta época, hubo una transformación de las funciones estatales con un acrecentamiento de la participación del Estado provincial en la gestión social de la población, sin embargo, dicha ampliación no fue acompañada de la creación de nuevas agencias destinadas a implementarlas. Salvo algunas iniciativas, no se instituyeron nuevas reparticiones orientadas al tratamiento de las nuevas problemáticas sociales, sino permanencias en los rasgos del organigrama provincial de los años veinte. A su vez, la trayectoria cordobesa, caracterizada por la creación incipiente de nuevas agencias estatales, puede contraponerse a otros gobiernos de provincia como el de Santa Fe, en donde existieron transformaciones más notables en la estructura estatal (Ortiz Bergia, 2015; Bacolla, 2016). En definitiva y, específicamente en el área social, debe entenderse que el sistema establecido entre las décadas de 1940 y 1950 fue producto de un proceso complejo previo, en el que se fueron instituyendo paulatinamente cambios en las competencias, en las estructuras estatales, en las relaciones interjurisdiccionales, en la distribución de recursos, etc. Estos cambios no fueron similares en todas las provincias, ya que existieron diferencias no sólo en la fisonomía que fue adquiriendo el Estado, sino también en los niveles de ejecución de las políticas públicas (Piazzesi, 2009; Bacolla, 2016; Ramacciotti, K. y Biernat, 2016).

Este contexto, en el que se evidencian distintos planos de avance de los Estados provinciales sobre la cuestión social, despierta nuestro interés por indagarlo sucedido en Mendoza, es decir, aquello que se refiere a las transformaciones institucionales y a las estrategias de intervención del gobierno provincial en el área sanitaria y asistencial llevadas a cabo para resolver las diferentes problemáticas (enfermedades infectocontagiosas, mortalidad infantil, etc.). Específicamente-con el fin de contribuir a ese conjunto de estudios provinciales a partir de la reconstrucción de la historia de un área estatal escasamente estudiada en la provincia-, nos enfocaremos en las reformas legislativas en materia de asistencia sanitaria y en la expansión de competencias, la creación (o no) de nuevas agencias estatales, el avance sobre otras jurisdicciones estatales, la relación con las entidades privadas de asistencia social, y la disponibilidad de recursos (humanos, materiales y económicos) que incidieron en la capacidad de acción del Estado provincial (Ortiz Bergia, 2016). Debe destacarse que, además de observar las transformaciones desde el punto de vista institucional y de distribución de recursos-siguiendo un discurso político que propiciaba mejorar y acrecentar los servicios ampliando y creando nueva infraestructura-, se valorarán específicamente aquellas políticas vinculadas con los planes de obra pública. Dichas políticas fueron significativas para los gobiernos de los años 30 no sólo desde el punto de vista económico, simbólico o estético, sino también porque estaban destinadas a mejorar las condiciones de vida de la población y lograr el bienestar social (Bellent, 2004; Jáuregui, 2013; Fernández, 2013, Piglia, 2014, Pettiti, 2016, Raffa, 2016).

\section{SALUD $\mathrm{Y}$ ASISTENCIA SOCIAL DURANTE EL NEOCONSERVADURISMO EN MENDOZA $(1932-1943)$}

Diagnóstico general y dificultades del Estado provincial para prestar servicios (1932-1935)

Durante los llamados gobiernos neoconservadores pueden identificarse claramente dos etapas en materia sanitaria, una fuertemente influida por el contexto político y económico de principios de la década y por el 
impacto de la crisis sobre los servicios de salud, y la otra favorecida por cierta recuperación económica y por reformas institucionales tendientes a mejorar las prestaciones. Dichas acciones reflejaron el avance del Estado provincial en materia sanitaria y asistencial.

Desde 1927 la industria vitivinícola, el principal motor de la economía provincial, venía experimentando una crisis de sobreproducción que se agravó en 1930. El deterioro de los salarios reales y el aumento del desempleo a nivel nacional, unidos a esta crisis local llevaron a una caída del precio del vino y la uva, hecho que afectó significativamente la economía provincial (Cerdá, 2011). Dicha crisis claramente repercutió en los presupuestos provinciales que bajaron entre 1932 y 1934 (Gráfico № 1), lo que incidió negativamente en la capacidad de la Dirección General de Salubridad de cumplir con sus funciones.

GRÁFICO No 1

Presupuestos de la Dirección General de Salubridad (1932-1940)

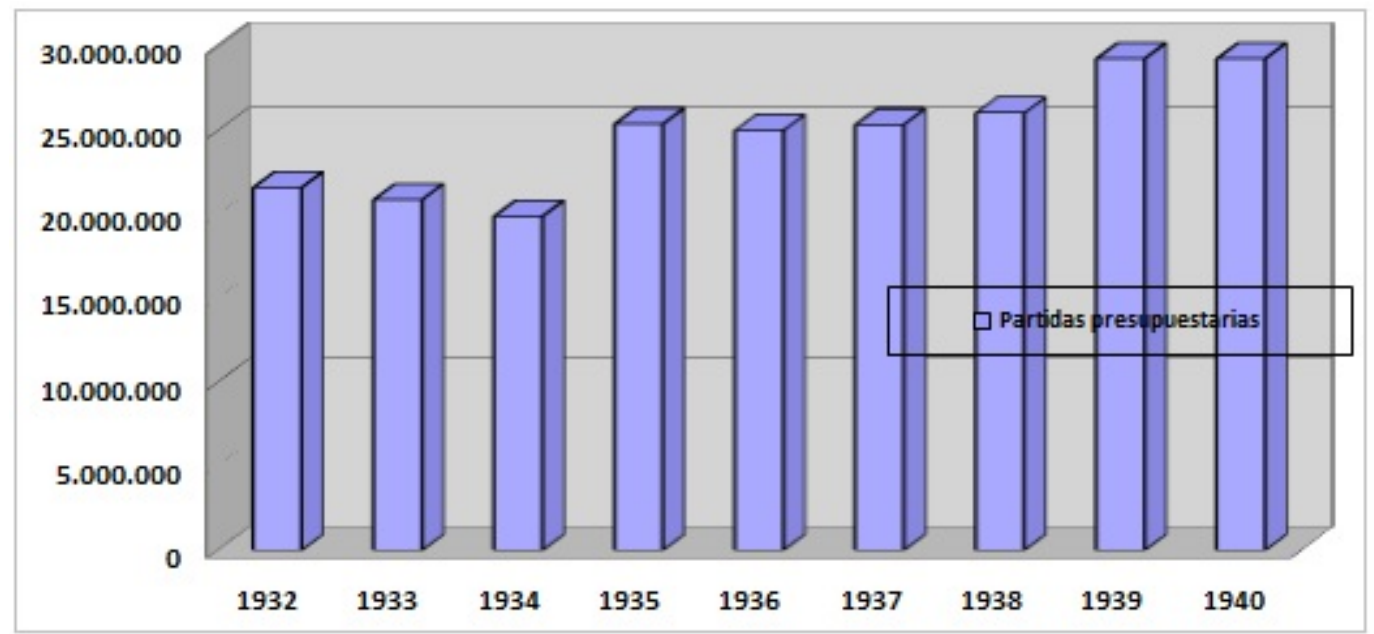

Fuente: Elaboración propia sobre la base de los datos de provincia de Mendoza,

Labor de Gobierno (1938-1941), gobernador Rodolfo Corominas Segura (s/p).

Según la ley de Sanidad No 926 de 1927, este máximo organismo sanitario, que a su vez dependía del Ministerio de Industrias y Obras Públicas, debía encargarse de todo lo relacionado con la profilaxis y la administración de dispensarios antivenéreos y antituberculosos de la provincia, como así también de la protección a la primera infancia, de los dispensarios de puericultura, y de la asistencia maternal a domicilio. Le correspondía también la inspección técnica de la higiene pública, vacunación, inspección de laboratorios químicos, profilaxis de las enfermedades infectocontagiosas, epidemias, policía sanitaria, droguería central y depósito de material médico. Debía proveer lo necesario a los hospitales y demás dependencias sanitarias, crear una escuela de enfermos y masajistas, inspeccionar baños, lavaderos públicos y farmacias. Es decir, se encargaba de todo lo relativo a la desinfección, inspección de hoteles, balnearios, casas de comercio, tambos, establecimientos industriales, higiene de las aguas en general, inspección de casas de prostitución, y reglamentación de la lactancia materna ${ }^{1}$.

Bajo su jurisdicción se encontraban los hospitales Emilio Civit, San Antonio, Carlos Ponce, Diego Paroissien y los regionales de San Martin, Rivadavia (zona Este), General Alvear y San Rafael (zona Sur). Completaban el mapa sanitario de la provincia las salas de primeros auxilios (provinciales y municipales), dispensarios y curatorios municipales, algunos establecimientos a cargo de asociaciones benéficas como el Hospital El Carmen (dependiente de la Sociedad San Vicente de Paul), y el dispensario para tuberculosos sostenido por la Liga Argentina contra la Tuberculosis ${ }^{2}$.

Durante los primeros años de la gestión de Ricardo Videla (1932-1935), los establecimientos provinciales tuvieron dificultades para cumplir con algunas de las funciones que le asignaba aquella ley de sanidad, y ello se reflejaba en la disminución de servicios, sobre todo en aquellos destinados a enfermos hospitalizados, servicios 
de ambulancia, vacunaciones (antidiftérica y antivariólica), desinfecciones, farmacia, como así también la cantidad de enfermeros, y enfermos atendidos por estos establecimientos (Gráfico No 2).

GRÁFICO No 2

Servicios prestados por la Dirección General de Salubridad y Asistencia Pública entre 1932 y 1937

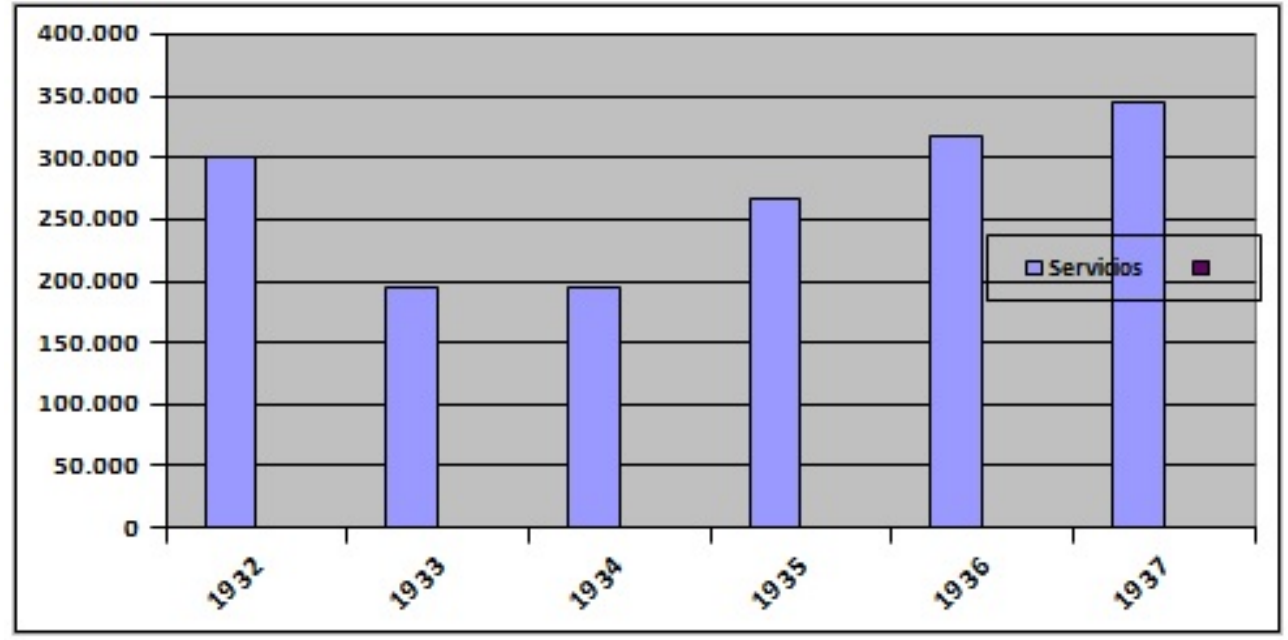

Fuente: Elaboración propia sobre la base de los Anuarios de la Dirección General de Estadísticas de la Provincia de Mendoza del año 1932-1937, Mendoza; Provincia de Mendoza, Labor de Gobierno Período 1938-1941, Gobernador Rodolfo Corominas Segura, Mendoza, Imprenta Oficial, s/p.

En este marco general, las tareas de vacunación y desinfección para prevenir y combatir enfermedades infecciosas como la difteria, la viruela y la tuberculosis en los diferentes departamentos de la provincia evolucionaron de manera diferente. En el Gráfico $\mathrm{N}^{\circ} 3$ puede verse que si bien el número de personas vacunadas contra la viruela aumentó levemente, disminuyó el número de personas que acudió a la vacunación antidiftérica. Si bien estas enfermedades no tenían mayor incidencia en los índices de mortalidad de la provincia, ocuparon siempre la atención de la prensa, la cual informaba casi diariamente sobre la existencia de enfermos y la situación en los diferentes departamentos. Con respecto a la difteria, por ejemplo, las cifras de mortalidad a causa de este mal, registradas por la Dirección General de Estadísticas y enviadas al Departamento Nacional de Higiene, daban cuenta de que en cuatro años la difteria se había multiplicado, y esto había aumentado la mortalidad entre 1929 y 1932 (de 35 casos a 181) ${ }^{3}$. Entre 1932 y 1934 la cantidad de personas con difteria habría disminuido, pero estos descensos no eran significativos ya que de 3.900 personas se pasó a 3.600 en esos años ${ }^{4}$. 
GRÁFICO Nº 3

Cantidad de Vacunas aplicadas entre 1932 y 1940

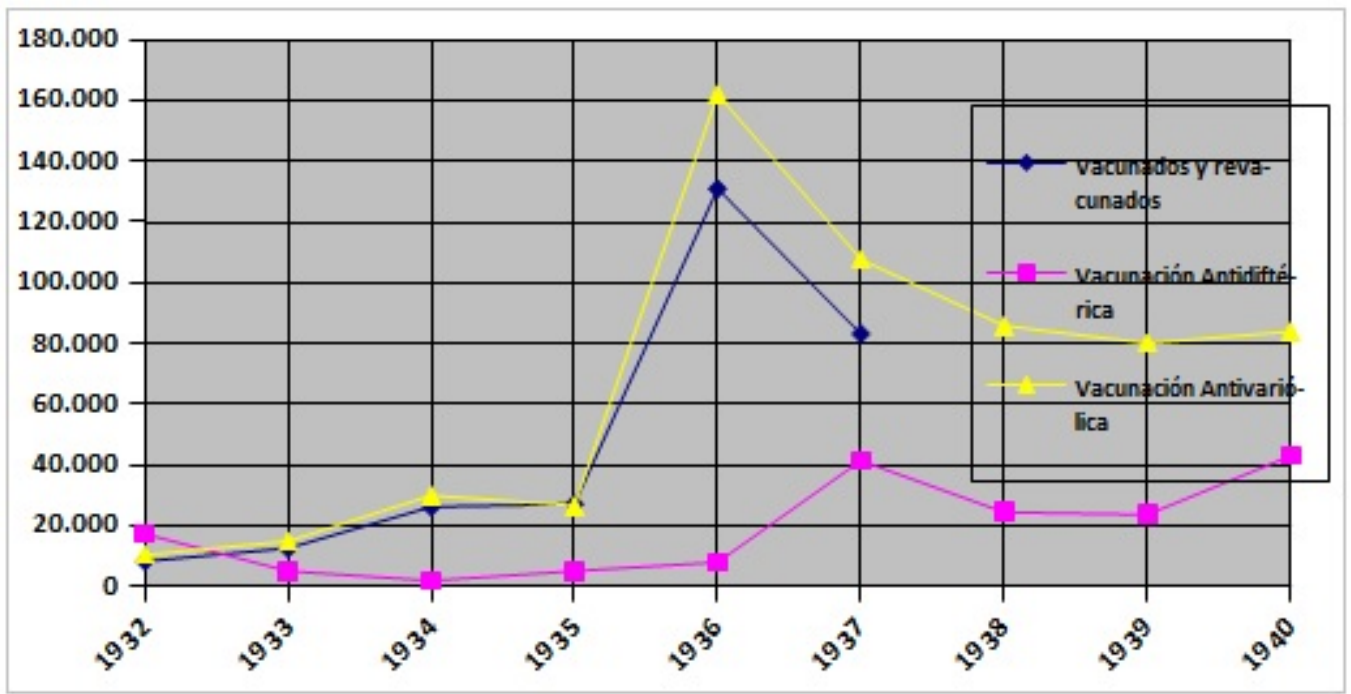

Fuente: Elaboración propia sobre la base de los Anuarios de la Dirección General de Estadísticas de la Provincia de Mendoza del año 1932-1937, Mendoza; Provincia de Mendoza, Labor de Gobierno Período 1938-1941, gobernador Rodolfo Corominas Segura, Mendoza, Imprenta Oficial, s/p.

Para la prevención y atención de enfermos infectocontagiosos, los gobiernos lencinistas construyeron el Hospital Néstor Lencinas en la Ciudad, el cual se especializaba en estas afecciones, como así también atendía a enfermos por tuberculosis y enfermedades venéreas (Richard-Jorba, 2012). A pesar de la existencia de este hospital, en la década de 1930 no existían los medios estatales suficientes para combatir la tuberculosis, ya que la provincia carecía de hospitales amplios con camas suficientes para cubrir las necesidades que demanda esta enfermedad. Es de destacar, sin embargo, el esfuerzo realizado por la Liga Argentina contra la Tuberculosis ${ }^{5}$, que cumplía sus funciones a través de los servicios prestados por su dispensario (revisaciones, inyecciones, análisis, recetas, etc.). Si bien esta entidad gozaba de cierta autonomía, en los hechos la falta de recursos actuó como condicionante a su accionar, y en 1932, ante la delicada situación económica que atravesaba-en parte por los retrasos de los aportes nacionales-, la institución comenzó a recibir el apoyo económico del Estado provincial, que le asignó por primera vez una partida en el presupuesto. Este tipo de acciones de asistencia social también se destinaron a otras instituciones privadas de carácter benéfico como los Asilos del Buen Pastor, de Mendigos y de Huérfanos, Monjas Dominicas de Guaymallén, Monjas Franciscanas, Hospital del Carmen de Godoy Cruz, Hospital de General Alvear, Centro Patriótico de Ayuda Social, Sociedad de Beneficencia de San Rafael y Asilo "Las Mercedes" de San Rafael. Todas ellas recibieron subsidios del Estado provincial $^{6}$. Es decir, en una primera etapa de graves dificultades económicas, el gobierno intervino en las acciones de las asociaciones benéficas mediante la distribución de recursos económicos, pero mantuvieron cierta independencia, ya que no llegó el Estado a absorber funciones o establecimientos de esta índole, a diferencia de otras provincias.

Sin embargo, a pesar de este apoyo inicial, en los años siguientes la Liga se vio obligada a reducir sus prestaciones debido a la disminución de más de un $50 \%$ de las subvenciones nacionales y provinciales, y a la menor cantidad de recursos recibidos en concepto de caridad pública ${ }^{7}$. No obstante, hacia 1935 logró revertir la situación y prestar mayores servicios respecto de 1932 (de 16.000 a 55.000 respectivamente) ${ }^{8}$. En el caso de otras provincias, como Córdoba, señala Carbonetti (2008) que la lucha contra la tuberculosis se caracterizó también por la falta de recursos estatales y de otros medios para combatirla, por lo que se trató de un problema sanitario que no tuvo solución inmediata. 
Las medidas de profilaxis y prevención también eran llevadas a cabo por los municipios, con recursos propios y personal municipal y, en ciertos casos, con profesionales solicitados a la Dirección de Salubridad 9 . Sin embargo, en la lucha antivenérea, por ejemplo, las comunas no siempre pudieron responder con sus propios recursos, algo que llevó al gobierno provincial a traspasar a su jurisdicción ciertos servicios municipales, como fue el caso del Curatorio antivenéreo "Benito Sicardi" perteneciente a la comuna de la capital ${ }^{10}$. Este cambio jurisdiccional, que significó la centralización de las funciones en manos del Estado provincial, marcó un antecedente del avance de la provincia sobre los municipios que luego se produciría con el peronismo. A fines de la década de 1940 se produjo el traspaso de las salas de primeros auxilios, con lo cual la provincia pasó a administrar cerca de 60 salas de las 22 que poseía anteriormente. Sin embargo, ese aumento de salas provinciales se debió también a la importante política de obra pública, tal como veremos luego. Según Carlos Evans, que gobernó la provincia entre 1952 y 1955, este traspaso se produjo con la intención de unificar el "comando sanitario" y poner en práctica la centralización de los servicios de atención médica, de manera de lograr un régimen uniforme y eficaz en calidad y cantidad de servicios. Se produjo así la misma tendencia que en otros territorios-como por ejemplo Córdoba-, por la cual se transfirieron a las provincias establecimientos que hasta entonces estaban bajo la órbita del municipio (Ortiz Bergia, 2012). Mediante la observación de las diferentes ediciones del diario Los Andes podemos decir que este avance sobre las comunas se debió principalmente a la falta de recursos municipales, que limitaba la capacidad de las mismas de prestar servicios, disponer de médicos, instrumental, material de curación, etc.

Por último, otra de las cuestiones a mencionar, la cual requería mayor intervención del Estado, era la mortalidad infantil, uno de los problemas más graves en Mendoza al comenzar la década de $1930{ }^{11}$. En los primeros años las prestaciones vinculadas a la protección de la maternidad y primera infancia, que incluían atención en consultorios de lactantes, régimen dietético (lactancia, alimentación), inyecciones y vacunación, consultorio obstétrico, inspección domiciliaria, recetas, cocina del lactante, etc., sufrieron la misma suerte que aquellas destinadas a la población en general, ya que pasaron de 417.189 en 1932, a 381.625 en $1934^{12}$.

\section{Reorganización institucional y AVANCES Del Estado Provincial (1935-1943)}

Acorde con la política llevada a cabo a nivel nacional, el proyecto político de los gobiernos neoconservadores intensificó la intervención sobre la asistencia social en las áreas de salud, educación y vivienda (Ver Raffa, 2017). Los debates y reflexiones emitidas en la Conferencia Nacional de Asistencia Social de 1933, realizada durante la presidencia de Agustín P. Justo, constituyeron para nuestro país un antecedente significativo, tanto en el proceso de profesionalización de la ayuda social como en la redefinición de la relación entre intervención estatal y asistencia pública. Impulsada por el ministro de Relaciones Exteriores Saavedra Lamas, se planteó que los problemas sociales debían ser tratados de un modo racional, metódico y científico, y que la intervención estatal debía orientarse hacia una mayor especificidad de lo social. A partir de una nueva legislación debían crearse nuevas modalidades de organización y coordinación técnica (mediante ficheros y guías de asistencia social; censos de obras de asistencia, beneficencia y cultura; Biblioteca Social, etc.). Se proponía, además, la coordinación interinstitucional y el debate acerca de las mejores respuestas para tratar enfermedades sociales, la problemática familiar, condiciones de trabajo, desocupación, y la incorporación de dimensiones no exploradas del desarrollo humano como el deporte y la recreación. Los propósitos de la Conferencia tuvieron por eje una mayor coordinación de la asistencia social oficial y privada para alcanzar la más alta eficiencia en los servicios de asistencia y protección del individuo, la familia y la colectividad, sin dispersión de energías ni superposición de obras(Krmpotic, 2002).

En el plano provincial, algunos avances comenzaron a evidenciarse a partir de mediados de la década, cuando el poder político reconoció que los problemas sanitarios provenían, en parte, de la falta de racionalidad técnica en la planificación y prestación de servicios, lo que derivó en un problema de distribución 
de recursos. Se trató de una situación que había sido señalada y reclamada -en reiteradas oportunidades- por la prensa local ${ }^{13}$. El escaso conocimiento de las verdaderas necesidades de las dependencias y de coordinación entre ellas era una de las fallas principales del sistema sanitario provincial, lo que llevaba a realizar gastos innecesarios, de los ya escasos recursos, por el retraso de subsidios nacionales ${ }^{14}$. Esta problemática fue explicada por el gobernador Guillermo Cano al fundamentar los cambios de orden técnico y administrativo que era preciso realizar:

Pienso que puede conseguirse alguna economía y mayor eficiencia en esta función pública (...), introduciendo un sistema más ordenado de gastos, tanto en la parte administrativa, como en la parte de consumos para los servicios médicos mismos. Hay, sin duda alguna, un poco de derroche, involuntario desde luego, que es menester evitar, para alcanzar resultados mejores, sin aumentar gastos.

Tengo el propósito de iniciar esta obra de reajuste, aplicando un sistema de estadística más moderno (mecánico), a fin de conocer y comparar cifras y poder corregir los errores, donde se hayan producido. Los señores médicos se distinguen por su celo profesional, colocando sus servicios al más alto nivel científico posible.

Solo falta un poco más de "sistema", para que la obra se perfeccione y rinda mejor, acercándose al ideal de su trascendente función social. Podemos estar orgullosos de nuestra organización sanitaria, pero debo, sin embargo, señalar una falla importante (...) Me refiero por una parte, a la exageración creciente con que el público quiere hacer uso de los servicios médicos gratuitos, excediendo muchas veces sus necesidades reales; y por otra, el abuso que significa a provechar los servicios del Estado, por las personas que están en condiciones de sufragarlos de su propio peculio. Con la primera causa se recarga innecesariamente el trabajo de los médicos, enfermeros, ambulancias y en general de todo el mecanismo de salubridad, sin esperar o sin tener un motivo real que lo justifique. La segunda causa recarga el esfuerzo de los médicos y el gasto de medicina y demás materiales, y toma lugar en los hospitales destinados a las clases humildes, desalojando injustamente a los que tienen mejor derecho para ser atendidos gratuitamente, porque no poseen recursos propios suficientes ${ }^{15}$.

En cuanto a las enfermedades infectocontagiosas, el gobierno mendocino había logrado, en esta segunda etapa, acentuar aquellas medidas de carácter profiláctico y de prevención, no sólo a través de campañas de vacunación (antidiftérica y antivariólica) y la desinfección de escuelas, locales, etc. en los distintos territorios de la provincia, sino también a través de la sanción de la ley que declaraba obligatoria y gratuita la vacunación antidiftérica preventiva para los niños de entre uno y doce años de edad ${ }^{16}$. La posibilidad obtenida por el Estado de intensificar aquellas medidas se debió, en parte, a los cambios en la asignación de recursos presupuestarios (Gráfico No 3), lo que le permitió subir las prestaciones (servicios de internación, vacunaciones, inspecciones, etc.) de cuestiones de mayor urgencia, tal como puede verse en el Gráfico $\mathrm{N}^{\circ} 2$ y Gráfico $\mathrm{N}^{\circ}$ 3. Con ello se logró disminuir las cifras de mortalidad infantil y mortalidad general. El nivel de ciertas enfermedades como la difteria se mantuvo constante, a pesar de las campañas de vacunación realizadas, incluso en el extremo sur de la provincia. En ello incidió la resistencia de los padres de vacunar a sus hijos en edad preescolar ${ }^{17}$.

Los aumentos en los presupuestos provinciales permitieron no sólo un incremento de servicios básicos destinados al binomio madre-hijo, que en 1936 llegaron a $450.000{ }^{18}$,sino también que fueran impulsadas nuevas políticas de asistencia social para una mayor protección a este sector de la población, como la creación de la Oficina de Servicio Social, agencia a cargo de las visitadoras de Higiene Social y Escolar ${ }^{19}$, egresadas de la escuela de reciente creación. Esta escuela, al igual que otras creadas en el país, se adecuaba a la idea de profesionalizar la ayuda social impulsada en la Conferencia Nacional de Asistencia Social de 1933, ya mencionada, de modo de "crear un ambiente social de orden y método científico en las obras de Asistencia, lucha contra la acción negativa de la Asistencia paliativa, promoviendo la organización de la preventiva y constructiva" (Solá y Becerra, 2009).

A través de la acción de las visitadoras de la Oficina de Servicio Social, la medicina preventiva y la sanidad escolar adquirían en Mendoza mayor impulso, y se hacían efectivas mediante diversas maneras. Una de ellas fue la atención de las mujeres que asistían a la Escuela de Madres, a partir de la proporción de asesoramiento y asistencia sobre puericultura, higiene y alimentación ${ }^{20}$. Por otra parte, la visitadora social designada en el Hospital de Niños (dentro del hospital Emilio Civit) concurría a los hogares de aquellos menores internados, 
de modo de conocer las condiciones de vida del paciente y elaborar informes y una historia clínica del niño bajo asistencia. En este sentido, la visitadora se convertía en colaboradora del médico. La Oficina colaboraba también con asociaciones de beneficencia como el Asilo de Huérfanos, la casa de Protección a la Madre Obrera y la Casa Cuna ${ }^{21}$, y realizaba tareas en el barrio de casas colectivas mediante un consultorio de medicina preventiva instalado para atender a niños en general y alumnos de la escuela barrial.

Aunque con diferentes matices, estos cambios institucionales que le otorgaron al Estado nuevas dimensiones y formas de intervención, se produjeron también en otras provincias. Cuando en Santa Fe fue creado el Departamento de Salud Pública, se incorporaron varias agencias estatales destinadas a la asistencia médica preventiva y curativa, y se abarcaron también varios aspectos específicamente sociales y poblacionistas como las oficinas de servicio social, sanidad escolar, higiene urbana y rural. Estas acciones fueron reforzadas por una agencia específica de educación, propaganda y divulgación sanitaria, un museo de higiene, biblioteca y publicaciones a través de diversos medios de comunicación (Bacolla, 2016; Rubinzal, 2006).

El mayor interés por parte del gobierno de Mendoza a prestar mayores y mejores beneficios al binomio madre-hijo puede verse también en la maternidad "José Federico Moreno" de capital, la cual prestaba servicio médico y social ${ }^{22}$. Los servicios obstétricos, que funcionaban divididos, fueron centralizados en dicha maternidad. Allí prestaban servicios las parteras de la asistencia pública y del Dispensario de Primera Infancia 23. La existencia del Servicio de Protección a la Maternidad y Primera Infancia y del Consultorio de niños de Segunda Infancia, como así también la incorporación de nueva infraestructura, como salas de maternidad y pabellones de niños en los hospitales de la provincia, fueron algunas iniciativas que llegaron a concretarse durante éste período.

Estas medidas, que requerían de recursos estatales, fueron posibles gracias a que se produjo una mayor racionalidad en los gastos. Se crearon algunos instrumentos que permitieron conocer las verdaderas necesidades de las dependencias y establecer orden de prioridades, tal el ejemplo de los inventarios sobre existencias de los establecimientos, el control de distribución de insumos por parte de la Droguería o Despensa Central ${ }^{24}$, y la designación de comisiones para realizar un sistema de estadística analítico que permitiera conocer la situación de cada establecimiento (hospitales, salas, etc.) en cuanto a los enfermos internados, personal de servicios, consumo de artículos, etc. Se solucionó además el problema de la atención de enfermos no urgentes en la asistencia pública que impedía prestar primeros auxilios de urgencia. Con el fin de descongestionar la afluencia de este tipo de pacientes (no urgentes) fue creado el servicio de Orientación de Enfermos, que los distribuía y derivaba hacia las distintas especialidades; se creó además un fichero general y una Oficia de Estadística Central. Por otra parte, se procedió reorganizar los consultorios externos de los hospitales, con el fin de prestar una eficaz atención a los enfermos no urgentes. Otra iniciativa fue la creación Comisión Asesora Ad-Honorem presidida por profesionales para estudiar el problema hospitalario ${ }^{25}$.

Por su parte, la ley de Ministerios No 1.294 de 1938, que creó el Ministerio de Gobierno y Asistencia Social, produjo un reordenamiento institucional muy importante pues significó una mayor asociación y coordinación de las cuestiones de salud y asistencia social, unificando asuntos que eran atendidos separadamente. Anteriormente, mientras las cuestiones laborales, la protección de los menores y niños, las subvenciones a instituciones de beneficencia, entre otros temas, estaban bajo la órbita del Ministerio de Gobierno. Todo lo concerniente a la Dirección General de Salubridad quedaba bajo jurisdicción de la cartera de Industrias y Obras públicas. Por lo tanto, un único ministerio absorbía lo relativo a regímenes o sistemas carcelarios y de establecimientos penales y correccionales, el Patronato de Liberados y Menores, la protección del sub-normal (sordomudo, ciegos, delincuente, abandonado, etc.), los reformatorios, asilos y colonias, la protección a la madre y al niño, la legislación del trabajo, el seguro obrero y el seguro social, la salubridad, los hospitales, salas de primeros auxilios, consultorios, dispensarios y servicios sanitarios en general y la policía sanitaria. Además, subvencionaba hospitales, asilos, colonias, o corporaciones de ayuda social ${ }^{26}$. 
Otra alternativa propuesta para dar mayor eficiencia y economía a la función sanitaria, tal como se había planteado también a nivel nacional (Biernat, 2016), fue el cambio en la estructura del sistema hospitalario caracterizado por la existencia de establecimientos dispersos, con escasa coordinación y conexión entre las distintas dependencias. Para nuestra provincia se propuso unificar y centralizar el comando sanitario, lo que a su vez llevaría a una descentralización de servicios (Mapa $\mathrm{N}^{\circ} 1$ ). Se formaron así dos núcleos básicos denominados centros sanitarios, uno con asiento en la capital y administrado por la Dirección General de Salubridad para los establecimientos del Gran Mendoza, zona Este y Norte de la provincia (oasis norte). El otro centro estaba ubicado en la ciudad de San Rafael, bajo la Subdirección General de Salubridad, y centralizaba los servicios de todo el Sur. A estos dos centros sanitarios (conectados entre sí), convergían las actividades de los hospitales inmediatamente próximos a los mismos, de las salas de primeros auxilios provinciales y de los consultorios de primera y segunda infancia (para el caso de la capital), de reciente creación. Quedaban fuera de este sistema las actividades de asistencia social de las entidades benéficas, con las cuales colaboraba mediante subsidios. Toda nueva obra pública que se crease debía formar parte de esta estructura e ir acompañada de un estudio racional de los servicios que prestara. Esta propuesta demandaba además una redefinición de la función y especialidades de los otros hospitales y establecimientos sanitarios ya existentes, como así también el cálculo anticipado de los gastos para prestar diferentes servicios.

Los establecimientos ubicados en la campaña se transformaban en "servicios de primeros auxilios o de preparación del paciente" y debían estar conectados a la vez con los centros de asistencia más complejos, que poseían todo lo indispensable para el mejor diagnóstico y tratamiento de los enfermos. Dicha conexión, sobre todo entre establecimientos separados por grandes distancias, se haría efectiva mediante el servicio aéreo de sanidad y policía, incluido en un proyecto de ley del año $1940{ }^{27}$, que además de proporcionar atención médica (regular y periódica) a las poblaciones ubicadas en zonas apartadas que carecieran de médicos y vías de comunicación, prestaba servicio de traslado de enfermos que requiriesen tratamientos urgentes o especializados. Sin embargo, esta iniciativa recién sería concretada en el año 1946,a partir de la ley provincial No $1.589^{28}$, que creaba el servicio denominado “Transporte Sanitario”, el cual consistía en un equipo aéreo para el traslado de enfermos o accidentados, personal técnico y elementos sanitarios en caso de epidemias. Aunque no tenemos certeza de las causas de este retraso, podemos conjeturar que se debió, en parte, a la falta de infraestructura (como por ejemplo pistas de aterrizaje en zonas inhóspitas de la provincia).

Tanto a nivel nacional como en provincias del Norte como Tucumán, la idea de comando sanitario único también fue diseñada, aunque nunca llegó a concretarse en su totalidad (Andrenacci, 2004; Belmartino, 2005; Biernat, 2016; Fernández y Rosales, 2016). En Mendoza desconocemos hasta qué punto pudo ponerse en práctica, aunque sabemos que fue una iniciativa adoptada -con algunos matices-por los gobiernos peronistas. Es decir, primero bajo la Dirección General de Salubridad, y luego del Ministerio de Salud Pública Provincial (creado en 1950), los servicios hospitalarios de la provincia debían quedar subordinados a un comando único. 


\section{MAPA $\mathrm{N}^{\circ} 1$}

Sistema Hospitalario dependiente del gobierno de la provincia.

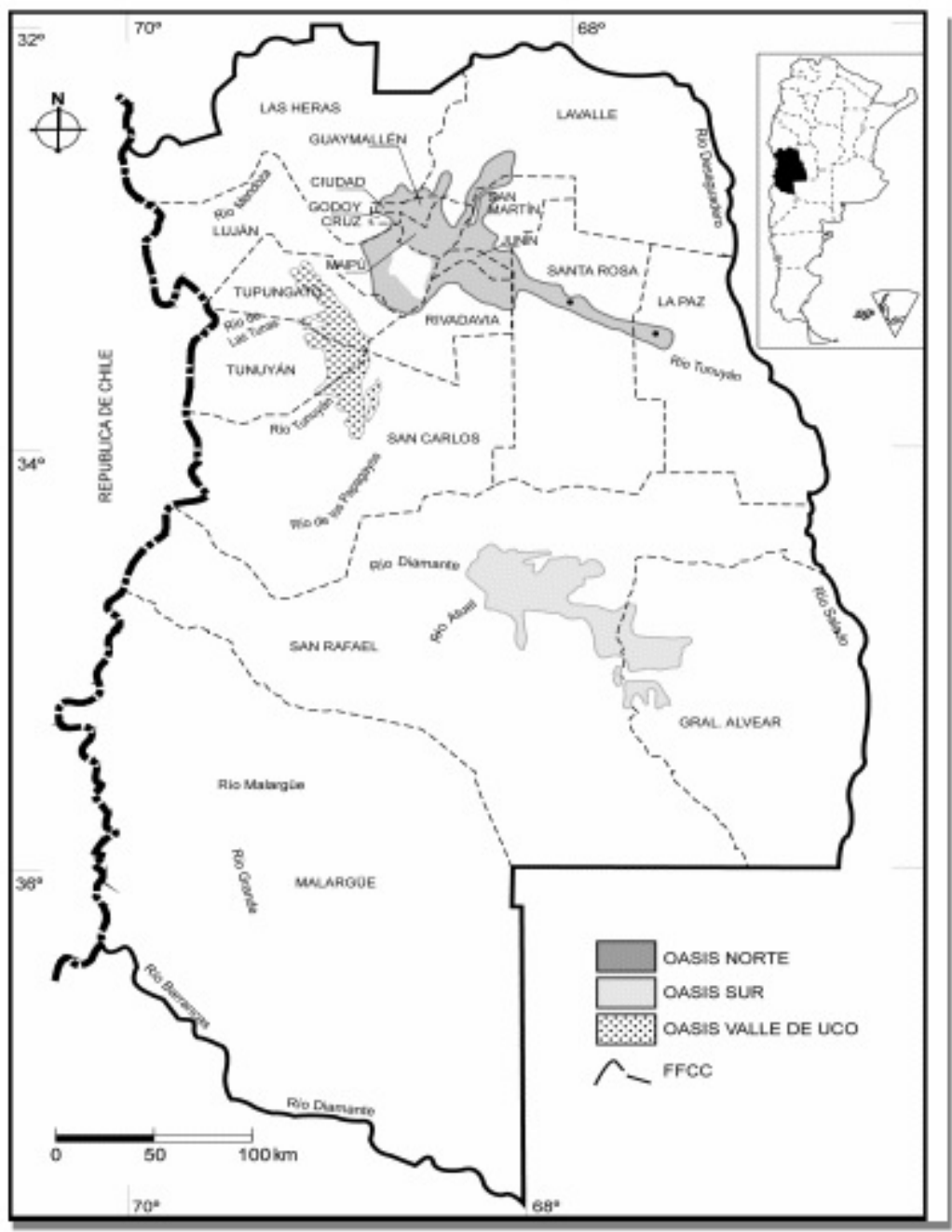

Fuente: Elaboración propia sobre la base de la Labor de Gobierno Período 1938-1941, gobernador Rodolfo Corominas Segura, Mendoza, Imprenta Oficial, s/p.

\section{Algunos AVANCES EN MATERIA DE OBRA PÚBliCA}

Tanto el gobierno nacional como los provinciales reaccionaron a la crisis agroexportadora, aplicando distintos mecanismos de regulación, poniendo atención en su mercado interno, modernizando y complejizando sus administraciones, y más tarde desarrollando industrias sustitutivas. Este perfil imprimiría el carácter progresista de la gestión neoconservadora, que se destacó por el emprendimiento de un volumen de obras públicas a escala provincial. Justamente, fue el desarrollo de equipamiento e infraestructura una de las acciones más representativas llevadas a cabo, pues buscó la disminución del desempleo y la reactivación económica a través de la atracción del capital extranjero y local al sector industrial. El ordenamiento de las finanzas provinciales y la prudencia en los gastos permitió destinar a obras públicas fuertes partidas en vialidad, obras de defensa contra aluviones y la construcción de edificios públicos; de todas ellas, más de la mitad fueron obras hospitalarias ${ }^{29}$. Es decir, aunque no ajenos a dificultades, los gobiernos neoconservadores se caracterizaron por una importante proyección de obra pública de carácter social destinada a mejorar las condiciones sanitarias de la población. Tal es así que, hacia 1940, un artículo del Diario Los Andes se titulaba 
"Su política de Asistencia Social fue una de las fases más interesantes de la gestión del Poder Ejecutivo", y en el cuerpo de la nota se refería a las construcciones y reparaciones hospitalarias ${ }^{30}$.

En lo que respecta a la salud, los planes giraron en torno a tres ejes principales: los hospitales urbanos, los hospitales regionales, y las salas de primeros auxilios en la campaña. Estas últimas estaban orientadas a expandir los servicios hacia las áreas rurales y llevar el auxilio médico a los habitantes que no podían trasladarse hasta los centros urbanos (ya fuera por distancia, por cuestiones de salud, o por los limitados recursos). En virtud de estos tres objetivos principales se incluyeron obras menores como reparaciones e instalaciones (cambios de cañerías, instalación de hornos incineradores de basura, etc.) en los distintos establecimientos, pero también otras de mayor envergadura que permitieran prestar mayores y mejores servicios e incorporar mayor cantidad de población al sistema(pabellones de niños, salas de maternidad, salas de cirugía, pabellones para crónicos, tuberculosos e infecciosos, entre otras).

No obstante, en virtud del mal estado de las finanzas provinciales y del retraso de los subsidios provenientes del gobierno nacional -característico de los primeros años-, la ejecución de varios trabajos públicos presentó dificultades. Esto incidió, en cierta medida, en la menor prestación de servicios señalada al comienzo del trabajo. Se produjo así una discontinuidad enla ejecución de ciertas obras de infraestructura, como en las ampliaciones de los hospitales Carlos Ponce, Rivadavia, San Rafael ${ }^{31}$ y Maipú ${ }^{32}$. Otro ejemplo fue el retraso en la construcción del Curatorio Antivenéreo Benito Sicardi en el Hospital San Antonio ${ }^{33}$, que, como mencionamos, fue traspasado a la provincia en 1933. El hecho de que en $1941 \mathrm{el} \mathrm{mismo} \mathrm{no} \mathrm{estuviera}$ terminado $^{34}$, hecho que afectaba la prestación de servicios, indica que la idea de centralización y avance sobre jurisdicciones menores no siempre tuvo éxito en la práctica.

Llegado el año 1938, ciertas dificultades financieras obligaron a revisar el plan de obras públicas que se ejecutaba "parcialmente", según el gobernador Corominas Segura, lo que paralizaba gran parte de las obras públicas proyectadas. Un año más tarde, el Poder Ejecutivo remitió a las cámaras legislativas un proyecto de ley que propiciaba un plan de obras a desarrollarse paulatinamente, y ajustado a las posibilidades financieras de la provincia. Dicho plan preveía en su conjunto las leyes $\mathrm{N}^{\circ} 1190,1216,1235$ y 1298 , que tenían principio de ejecución, pero las modificaba y unificaba para darles una estructura orgánica. De esta manera se resolvían las dificultades producidas en el cumplimiento de aquellas leyes, por la falta de negociación de títulos para la terminación de las obras comenzadas y por la insuficiencia de los créditos máximos permitidos para cada una. La nueva ley, sancionada bajo el $\mathrm{N}^{\circ} 1351$, preveía entonces los fondos necesarios para cubrir estas insuficiencias de créditos, pagar obras ejecutadas, terminar las iniciadas, concluir los estudios de construcciones comprometidas, autorizar el pago de expropiaciones y disponer la construcción de determinadas obras ${ }^{35}$.

Dado este conjunto de medidas relacionadas con la financiación de obras, y una vez lograda la participación del gobierno de la nación a través de subsidios, se construyeron finalmente 5 salas de primeros auxilios 36 , las que se sumaban a obras (de mejoramiento y habilitación) realizadas en años anteriores ${ }^{37}$. A las aproximadamente 25 salas de primeros auxilios existentes en 1941 debían agregarse 17 edificios más, aunque esto no logró concretarse durante éste gobierno. Varias de ellas continuaron funcionando en las escuelas o en locales alquilados, a pesar de que la prensa destacaba la insuficiencia de asistencia social en varios departamentos de la provincia ${ }^{38}$. Algunas de las obras quedaron sólo en los proyectos, y fueron concretadas posteriormente con el peronismo.

Si bien problemas económicos llevaron al retraso, paralización y cierto incumplimiento de los planes trazados, es indudable que la provincia pudo contar con nueva infraestructura (diversos pabellones, salas de cirugía, entre otras); su obra de mayor envergadura fue un hospital de escala microregional, el Central. Según Cecilia Raffa (2016), el mismo significó desde el punto de vista arquitectónico un avance en la concepción compositiva de los hospitales en Mendoza, propuesta técnica que apuntaba hacia la modernización hospitalaria. Las grandes expectativas que generó en el sector político este hospital, en tanto 
daba solución al problema hospitalario al menos en parte, hizo que el gobierno tomara medidas para su pronta puesta en funcionamiento ${ }^{39}{ }^{40}$. No obstante ello, fue habilitado provisoriamente por la intervención federal del coronel Vargas Belmonte en 1944.

\section{A MODO DE SÍNTESIS}

El presente trabajo es un aporte a la historia del Estado "desde adentro", un Estado visto desde la dinámica de sus agencias y de sus políticas con una perspectiva de abordaje en la que predomina lo micro, ya que, enfocado en la provincia de Mendoza, evidencia las particularidades del sistema sanitario local. Se lograron mostrar aquí, no sólo las potencialidades o los avances del aparato estatal, sino también sus dificultades, incapacidades o limitaciones para responder a distintas problemáticas, impuestas tanto por condicionamientos internos como externos. Es decir, se intentó mostrar tanto lo que hizo el Estado como aquello que no pudo hacer o los lugares a los que no logró llegar (Bohoslavsky y Soprano, 2010).

En los primeros años de la década de 1930 existieron límites en la capacidad del Estado para dar respuesta a las necesidades sanitarias de la población. Los obstáculos para hacer frente a las distintas problemáticas sociales se vincularon principalmente con los efectos de la crisis de 1930, pero también con la desorganización técnica y administrativa del sistema, gastos innecesarios, improvisaciones, y las menores posibilidades de financiamiento para la prestación de servicios. La obra pública de carácter médico asistencial tuvo una importancia fundamental, aunque cuestiones económicas incidieron también en la posibilidad de llevar a cabo esta política de manera continua y sin interrupciones. A pesar de ello, la provincia logró contar con mayor infraestructura, sobre todo a finales de la gestión, lo que se evidenció también en una expansión en materia de servicios sanitarios y asistenciales.

Si bien la transformación sustancial en el organigrama sanitario se produjo con la creación del Ministerio de Salud Pública Provincial (en 1950), y el sistema de salud adquirió otro matiz y mayor intensidad en ciertas cuestiones, adherimos a lo sostenido por la historiografía provincial de que dicha transformación fue producto de un proceso previo (Biernat y Ramacciotti, 2012), y que heredó prácticas de los años 30, diseñadas para mejorar lo prestación de servicios, realizar economía en los gastos e incluir mayor cantidad de población al sistema, tal el caso de la provincialización de establecimientos municipales, una mayor expansión hacia la dimensión social, la coordinación y unificación de los servicios sanitarios, y la expansión de la obra pública.

\section{REFERENCIAS}

Andrenacci, L. et. al. (2004). Acerca del Estado de Bienestar en el peronismo clásico (1943-1955), En J. Bertranou et al., El pais del no me acuerdo. (Des)memoria institucional e historia de la politica social en Argentina, pp. 83-114. Buenos Aires: Prometeo Libros.

Armus, D. (2007). La ciudad impura. Salud, tuberculosis y cultura en Buenos Aires, 1870-1950. Buenos Aires: Edhasa.

Bacolla, N. (2016). Nuevas capacidades estatales para una sociedad transformada: Instituciones y políticas sanitarias en la provincia de Santa Fe primera mitad del siglo XX. Trabajos y Comunicaciones, 44, pp. 1-25.

Ballent, A. (2004). La red nacional de caminos y la construcción de lugares turísticos en la década de 1930. Ponencia presentada al Taller Internacional Desplazamientos, Lugares, Identidades, 11-13 mayo 2004. Buenos Aires.

Belmartino, S. (2005). La atención médica argentina en el siglo XX. Instituciones y procesos. Buenos Aires: Siglo Veintiuno.

Biernat, C., y Ramacciotti, K. (2012). Politicas sociales, entre demandas y resistencias. Argentina 1930-1970. Buenos Aires: Biblos.

Biernat, C. (2016). Continuidades y rupturas en el proceso de centralización de la administración sanitaria argentina (1880-1945). Trabajos y Comunicaciones, 44, pp. 1-23. 
Bohoslavsky, E. y Soprano,G. (2010). Una evaluación y propuestas para el estudio del Estado en Argentina. En Un Estado con rostro humano. Funcionarios e instituciones estatales en Argentina (desde 1880 a la actualidad), pp. 9-55. Buenos Aires: Prometeo Libros y Universidad Nacional General Sarmiento.

Carbonetti, A. (2008). Un plan para combatir la tuberculosis en Córdoba en la década del '30. Salud colectiva,(4) 2, pp. 203-218.

Cerdá, J. M. (2011). Condiciones de vida y vitivinicultura. Mendoza, 1870-1950. Bernal, Provincia de Buenos Aires. Universidad Nacional de Quilmes.

Fernández, N. (2013). Construir la provincia. Estado, Politica y Obras Públicas en el gobierno de Manuel Fresco, 1936-1940 (Tesis para la Maestría en Ciencias Sociales con Mención en Historia), Universidad Nacional de Luján, Buenos Aires, Argentina.

Fernández, M. E., y Rosales M. del C. (2016). Trayectorias locales y proyecciones nacionales en el proyecto de centralización sanitaria en Tucumán (1900-1950). Trabajos y Comunicaciones, 44, pp. 1-24.

Girbal-Blacha, N. (2003). Mitos, paradojas y realidades de la Argentina peronista (1946-1955). Una interpretación histórica de sus decisiones politico-económicas. Bernal: UNQ.

Hirschegger, I. (2010). El bienestar social durante el peronismo clásico. La expansión de los servicios de salud hacia las áreas rurales de los municipios de la provincia de Mendoza (1946-1955). Mundo Agrario, pp. 1-39.

Krmpotic, C. (2002). La Conferencia Nacional de Asistencia Social de 1933. Los debates en torno al progreso, la pobreza y la intervención estatal. Scripta Ethnologica, XXIV, pp. 1-22.

Jáuregui, A. (2012-2013). Obras Públicas y eficiencia burocrática (1932-1943).Travesía, 14-15, pp. 79-106.

Ortiz Bergia, M. J. (2012). La construcción del Estado social en el interior Argentino. Cambios y continuidades en las Politicas sociales en Córdoba, 1930-1955 (Tesis de Doctorado en Historia), Universidad Nacional de Córdoba, Argentina.

Ortiz Bergia, M. J. (2012). Provincia y Nación en el proceso de construcción de un Estado centralizado. Políticas sanitarias en Córdoba (1943-1946). Quinto Sol. Revista de Historia Regional, 16, 1-23.

Ortiz Bergia, M. J. (2015). La compleja construcción del Estado intervencionista. Lógicas políticas en la conformación de una estructura estatal provincial, 1930-1955. Trabajos y Comunicaciones, 42, pp. 1-15.

Ortiz Bergia, M. (2016). La centralización estatal en la Argentina y el sistema de salud público cordobés en la primera mitad del siglo XX. Trabajos y Comunicaciones, 44, pp. 1-17.

Ospital, S. (2009). Modernización estatal y regulación económica en provincias vitivinicultoras. Mendoza, 1936-1946. Mundo Agrario18, pp. 1-14.

Petitti, M. (2016). Educación y obra pública durante el primer peronismo. La construcción de escuelas en la provincia de Buenos Aires. Anuario del Instituto de Historia Argentina, 16(1), pp. 1-17.

Piazzesi, S. (2009). Conservadores en Provincia. El iriondismo santafesino. 1937-1943. Santa Fe, UNL.

Piglia, M. (2014). Automóviles, turismo y caminos. Los clubes de automovilistas y la formación de las políticas turisticas y viales en la Argentina (1918-1955). Buenos Aires. Siglo XXI.

Raffa, C. (2016). Propuestas técnicas y prácticas políticas: Arquitectos en la Dirección de Arquitectura (Mendoza, 1932-1955). En F. Rodríguez Vázquez y C. Raffa, pp. 84-111, Profesionalizando un Estado provincial, Mendoza. 1890-1955. Mendoza: Instituto de Historia Americana y Argentina. F. Filosofía y Letras. UNCuyo.

Raffa, C. (2017). Arquitectura Pública y Asistencial Social: obras para el amparo de menores (Argentina, 1932-1943). En Coordenadas. Revista de historia local y regional Vol. 5, pp. 22-38.

Ramacciotti, K. (2010). Administración sanitaria: reformas y resultados obtenidos, Argentina (1946-1955). En E.Bohoslavsky y G. Soprano (editores), Un Estado con rostro humano. Funcionarios e instituciones estables en Argentina (desde 1880 hasta la actualidad), pp. 181-222. Buenos Aires: Universidad Nacional General Sarmiento.

Ramacciotti, K. y Biernat, C. (coord.) (2016). Dossier, Nación, provincias y municipios en la centralización del sistema sanitario argentino, 1900-1955.Trabajos y Comunicaciones, 44, pp. 1-9

Richard-Jorba, R. (2012). Los gobiernos radicales de los lencinas en Mendoza. Avances del Cesor, VIII, pp. 31-62. 
Rubinzal, M. (2006). Volviendo a los orígenes del servicio social. El caso de la Escuela de asistentes sociales de Santa Fe. Avances del Cesor, 15, pp. 113-134.

Solá, M., y Becerra, N. (2009). Intervención Social en la Argentina de los años 30: La profesionalización de la asistencia social. Historia Caribe, 15, pp. 139-157.

\section{Notas}

1 Ley No 926, provincia de Mendoza, Boletín Oficial, 6 de diciembre de 1927.

2 Además, existían otras instituciones benéficas que realizaban asistencia social en la provincia como el Asilo de Huérfanos, la Casa Cuna, el Asilo de Mendigos, las Monjas del Buen Pastor, las Monjas Dominicanas de Guaymallén, las Monjas Franciscanas (enfermeras a domicilio), el Centro Patriótico de Ayuda Social, entre otras.

3 Diario Los Andes, 16 de marzo de 1933, p. 5; 17 de marzo de 1933, p. 5.

4 Informe Anual sobre la marcha de la Administración Pública, Período 1935-1936 del gobernador de la Provincia Dr. Guillermo Cano a la Honorable Legislatura, Mendoza, 1936, p. 147.

5 Según Armus (2007), en su estudio sobre esta enfermedad en la ciudad de Buenos Aires, la Liga fue la referencia institucional más influyente y perdurable de la sociedad civil, ya que logró consenso acerca de la urgencia de combatir la enfermedad, lo que se concretóa partir de 1935 con la Primera Cruzada Nacional Antituberculosa.

6 Provincia de Mendoza, Mensaje del gobernador Ricardo Videla a la Legislatura, 1934, p. 81.

7 La institución contaba con aproximadamente 200 socios (médicos, particulares, etc.) que contribuían a su sostenimiento mediante la organización de colectas y fiestas de beneficencia que en años anteriores habían sido una medio eficaz de ayuda para la institución. En el año 1933 el producto de dichas colectas, como así también de las cuotas de los socios, habría sufrido grandes disminuciones (Los Andes, 1 de enero de 1933, p. 4).

8 Los Andes, 1 de enero de 1933, p. 4; Informe Anual sobre la marcha de la Administración Pública, Período 1935-1936..., op. cit., p. 111.

9 Los Andes, 18 de marzo de 1933, p. 5; 21 de marzo de 1933, p. 12.

10 Los Andes, 11 de enero de 1933, p. 4; 11 de febrero de 1933, p. 5.

11 Provincia de Mendoza, mensaje del gobernador Ricardo Videla a la Legislatura, 1934, p. 19.

12 Anuario Estadístico de 1932, op. cit. p. 180; Anuario Estadístico de 1934, op. cit., p. 220.

13 Diario Los Andes, 19 de agosto de 1934, p. 5; 8 de diciembre de 1934, p. 5.14 de julio de 1936, p. 5; 17 de julio de 1936, p. 5.

14 Ministerio de Industrias y Obras Públicas, Decreto N 53, 29 de enero de 1937

15 Informe Anual sobre la marcha de la Administración Pública, Período 1935-1936 del gobernador de la provincia Dr. Guillermo Cano a la Honorable Legislatura, Mendoza, 1936, p. 47.

16 Ley $N^{\circ}$ 1177, Boletín Oficial de la provincia de Mendoza, 21de abril de 1936.

17 Mensaje del gobernador a la Legislatura, Mendoza, 1939, pp. 73-74.

18 Anuario Estadístico de la Provincia de Mendoza, Mendoza, 1937, p. 248.

19 Informe Anual..., op. cit.1936, p. 225; Labor de Gobierno Periodo 1938-1941..., op. cit., p. 39.

20 Informe Anual sobre la marcha de la Administración Pública, Periodo 1935-1936..., op. cit., p. 224.

21 Informe Anual..., op. cit. 1936, p. 225; Labor de Gobierno Periodo 1938-1941..., op. cit., p. 39.

22 Incluía asesoramiento, gestiones en defensoría de menores, colocación de madres y de niños, etc.

23 Mensaje del gobernador Corominas Segura, op. cit., 1939, p. 73.

24 Ministerio de Industrias y Obras Públicas, Decreto $N^{\circ} 53,29$ de enero de 1937.

25 La misma estaba integrada por el director Salubridad, Dr. Félix Aguinaga, los Dres. Alfredo Metraux y Pedro Calderón, el director de Arquitectura, Arq. Arturo Civit, y el contador general de la provincia, Sr. José Nudo (provincia de Mendoza, Labor Gubernativa, Gobernador Adolfo Vicchi, 1941-1944, pp. 29-31).

26 Provincia de Mendoza, Boletín Oficial, 14 de enero de 1939.

27 Mensaje del Gobernador a la Legislatura, Mendoza, 1940, p. 267.

28 En Boletin Oficial, 16 de diciembre de 1946, p. 6168.

29 Provincia de Mendoza, Mensaje del gobernador Ricardo Videla a la Legislatura, 1934, p. 13.

30 Diario Los Andes, 4 de enero de 1940, p. 5.

31 Mensaje..., 1934, op. cit., p. 111

32 Los Andes, 1 de enero de 1936, p. 13.

33 Mensaje..., 1936, pp. 226-227.

34 Labor de Gobierno..., op. cit., 1938-1941, s/p.

35 Boletín Oficial de la Provincia de Mendoza, 30 de setiembre de 1939. 
36 En Uspallata, Los Arboles (Tunuyán), Las Catitas (Santa Rosa), Cosa de Araujo (Lavalle) y Tupungato (Diario Los Andes, 1 de enero de 1940, p. 4).

37 Mejoras en Tupungato y Malargüe, habilitación en Villa Atuel y Monte Comán de San Rafael.

38 Diario Los Andes, enero de 1940.

39 Entre ellos proyectos de ley solicitando fondos para la puesta en funcionamiento y la ampliación y terminación del edificio (Los Andes, 7 de mayo de 1942, p. 6; 15 de mayo de 1942, p. 5).

40 Los Andes, 25 de enero de 1944, p. 7. 